\title{
Efectos de un programa de ciberconvivencia en la prevención del cyberbullying
}

\author{
Jesús POZAS RIVERA, Tania MORALES REYNOSO, Rocío MARTÍNEZ-VILCHIS
}

\author{
Universidad Autónoma del Estado de México, México
}

(Recibido el 4 de Abril, 2018; Aceptado el 22 de Mayo, 2018)

RESUMEN: Con la utilización de los entornos virtuales como medios de socialización, problemáticas como el bullying se han trasladado al ciberespacio. A pesar de que el ciberbullying es una problemática actual dentro de las instituciones educativas, pocas son las propuestas de programas que buscan que los menores aprendan a utilizar y convivir de manera adecuada en los entornos virtuales. El objetivo general de esta investigación es que los estudiantes muestren un comportamiento adecuado en las relaciones interpersonales que tienen en los entornos virtuales a través de la puesta en marcha de un programa basado en la ciberconvivencia, y con ello, una disminución del acoso escolar cibernético. La evaluación de la efectividad de dicho programa se realizó por medio de un diseño cuasi experimental pretest-postest, contando con un grupo control sin exposición al programa $(\mathrm{N}=44)$ y un grupo experimental con exposición al mismo $(\mathrm{N}=44)$ de alumnos de bachillerato con edades entre 15 y 18 años. Entre los resultados obtenidos, se destaca la disminución de la victimización y justificación del cyberbullying en el grupo experimental y manteniéndose en el grupo control.

Palabras clave: cyberbullying, ciberconvivencia, prevención.

\section{Effects of a cyber-coexistence program on prevention of cyberbullying}

\begin{abstract}
ABSTRAC: With the use of virtual environments as means of socialization, problems such as bullying have been transferred to cyberspace. Although cyberbullying is a current problem within educational institutions, there are few proposals for programs that seek to enable children to learn to use and coexist in an adequate way in virtual environments. The objective of this research is that students show an appropriate behavior in the interpersonal relationships they have in virtual environments through the implementation of a program based on cyber-survival, and with it, a decrease in cyber bullying. The evaluation of the effectiveness of this program was carried out by means of a quasi-experimental pretest-postest design, with a control group without exposure to the program $(\mathrm{N}=44)$ and an experimental group with exposure $(\mathrm{N}=44)$ Of high school with ages between 15 and 18 years. Among the results obtained, we highlight the decrease in victimization and justification of cyberbullying in the experimental group and remaining in the control group.
\end{abstract}

Keywords: cyberbullying, cyber-coexistence, prevention. 
Correspondencia: Jesús Pozas Rivera. Facultad de Ciencias de la Conducta. Universidad Autónoma del Estado de México. Filiberto Gómez s/n, Toluca, México, C.P: 50100. Tel. 7221111860. Email: pozas.jesus_psic@outlook.com.

\section{Introducción}

El contexto actual en el que las personas se desarrollan ha sido altamente influenciado por un conjunto de cambios ocasionados por las tecnologías de la información y comunicación (TIC) que afectan desde ámbitos generales como la globalización hasta llegar a entornos más cercanos como lo son el escolar y familiar (Programa Aprende de la Junta de Castilla y León, 2011; Belloch, s.f.); esto trae consigo la aparición de una sociedad 2.0 o sociedad de la información que se encuentra hiperconectada, la cual responde la necesidad de organizar la información existente y el cómo se transmite, dando paso a una nueva forma de sociabilización por medio de estas tecnologías (Moravec, 2011), siendo la manera más notable la interacción a través de redes sociales.

Tan solo en México en el año 2015 existían entre 65 y 68 millones de internautas lo cual representa aproximadamente a un $59 \%$ de la población total del país; de este total de internautas un $34 \%$ de los internautas mexicanos pertenecía a un rango de edad menor a 18 años y presentaban un promedio diario de conexión de 7 horas con 14 minutos (AMIPCI, INFOTEC y Elogia, 2016; iab México, Televisa.com y Millward Brown, 2016). Esto demuestra el alcance del uso de las TIC en la población y la importancia que tiene en la manera en la que las personas se comunican entre sí.

Uno de los efectos de la democratización del internet se observa en la interacción entre los más jóvenes y los adolescentes, siendo ellos los usuarios principales que han incorporado los ambientes virtuales a su cotidiano, lo que incluye también la violencia entre iguales (Del Rey y Ortega, 2007; Moreno, 2001), trasformando el fenómeno del acoso escolar en nuevas manifestaciones que reciben el nombre de cyberbullying.

Éste término hace referencia al acto en que un individuo o grupo utiliza el internet $\mathrm{u}$ otras TIC con la finalidad de dañar, a través de una conducta deliberada, repetitivita y hostil, esta conducta conlleva crueldad social (Belsey, 2005; Willard, 2005; Buelga y Pons, 2012; Calmaestra, 2011); dicho acto se realiza hacia una víctima la cual no puede defenderse adecuadamente (Smith, Mahdavi, Carvalho, Fisher, Russell y Tippett, 2008), y para ser considerado como tal debe de darse entre compañeros de escuela (niños o adolescentes) (Aftab, 2010; Garaigordobil, 2011). Dichos comportamientos violentos adquieren distintas formas o tipos (Aftab, 2010; Kowalski, Limber y Agatston, 2008).

No todos los actos de violencia se consideran como cyberbullying. Los actos que derivan de la ingenuidad o falta de competencias digitales por parte de uno de los involucrados no pueden ser considerados como tal. Así mismo la repetición en un sentido estricto, para el caso del acoso escolar cibernético, tampoco es un indicador, debido a que no hay forma de medir el número de veces que se realizó la agresión.

Es por eso que el cyberbullying es un fenómeno mucho más complejo que el bullying tradicional, aunque comparte algunas de sus características como son: el desequilibrio de poder psicológico o social, la intencionalidad de causar daño y que se presenta exclusivamente entre pares. Sin embargo, las diferencias entre los dos tipos de acoso radica en el perfil anónimo del victimario, en la desaparición del tiempo y espacio de los ataques (pueden realizarse en (C) Psy, Soc, \& Educ, 2018, Vol. 10(2) 
cualquier lugar y horario, sin distinción de contexto familiar, o escolar) y su capacidad para volverse privado, cuando se hace de forma directa entre los implicados, o público, cuando otro usuarios pueden acceder a estas situaciones de violencia (Ortega, 2015). Y por esta razón se convierte en un tipo de fenómeno de mayor complejidad.

En el caso de México, existen investigaciones que intentan determinar la prevalencia de cyberbullying que existe en población adolescente (Del Río, Bringué, Sádaba y González, 2009; García-Maldonado et al., 2012; Lucio, 2009); y para el caso específico de la población de bachillerato de la Universidad Autónoma del Estado de México, Martinez-Vilchis y colaboradores (2015), presentaron un estudio en el cual la prevalencia de cyberbullying reportada en una muestra significativa de 637 alumnos fue de un $36.1 \%$ con una frecuencia incidencia variable, tomando en cuenta los roles de víctima, victimario y observador; de manera específica, la prevalencia de víctimas fue de un $23.86 \%$, la de victimarios fue de un $7.69 \%$ y la de observadores de un $67.66 \%$.

Actualmente en materia de prevención del cyberbullying existen programas que han reportado tener efectividad como lo son el programa propuesto por Palladino, Nocentini y Menesini (2012), el programa ConRed (Del Rey, Casas y Ortega, 2012) y el Cyberprogram 2.0 (Garaigordobil y Martínez-Valderrey, 2015), resultando ser pocos para una problemática tan masificada como lo es el cyberbullying.

Tomando en cuenta que la violencia nace con la intencionalidad de dañar al otro para obtener algún tipo de gratificación o beneficio (Vernieri, 2010); y que la interacción virtual que se da a través una red social surge a partir de la necesidad natural de toda persona de relacionarse y como resultado aparecen conexiones reales y cotidianas que se forman de manera orgánica (Christakis y Fowler , 2010), es necesario implementar programas de prevención que estén enfocados a cuidar que esta sociabilización se dé una manera armónica.

Es en este encuadre donde parecen conceptos íntimamente ligados a la convivencia en la red como son: la ciudadanía digital y la ciberconvivencia.

Por ciudadanía digital se entiende el buscar que los internautas sean capaces de usar y criticar los medios digitales como base para crear una responsabilidad cívica que lleve a una humanización de las tecnologías por medio de la tolerancia, el dialogo y la colaboración. Es decir, se transpolan al mundo digital aspectos como la educación, derechos, riegos, normas de etiqueta, reglas, leyes, identificación social y demás conjunto de conceptos que se comprenden dentro de la ciudadanía en los entornos presenciales (Galindo, 2009; Meneses, 2012; Fundación UNAM, 2013).

Hablar de ciudadanía digital, es referirse de manera amplia a la necesidad de formar pautas de convivencia con la finalidad de tener un ambiente seguro más a la hora de interactuar en internet, esto se da por medio de acciones y actitudes que procuran desarrollar competencias ciudadanas, valores y habilidades para la vida; pero esto no es fácil ya que el concepto de ciudadanía digital aún se está definiendo lo cual ocasiona una difícil intervención ya que la formación de ciudadanos es un proceso por sí mismo laborioso y no se tiene indicadores claros del progreso que se llega a lograr (Flores-Fernández, 2009; Flores-Fernández, 2010).

Es esta dificultad de abordar la ciudanía digital en su totalidad lo que encamina a focalizarse sobre aspectos mejor delimitados para inculcar el uso adecuado de las TIC y prevención de la violencia virtual. Es en este sentido que podríamos hablar del segundo término. 
La ciberconvivencia se entiende como una nueva dimensión de convivencia la cual llevan a cabo los adolescentes por medio de las TIC tomando en cuenta las relaciones interpersonales que desarrollan con su red de iguales por medio de los entornos virtuales, contemplando factores como la calidad de estas relaciones, las reglas que las rigen las cuales deben ser conformadas democráticamente y el proceso instruccional que los lleva interactuar; así como las conductas y emociones que surgen en esta interacción (Ortega, 2011; CastroSantander, 2012; Castro-Santander, 2013; Ortega, Casas y Del rey, 2014; Ortega, 2015).

En lo que respecta a programas diseñados para mejorar la ciberconvivencia encontramos propuestas implementadas en España como lo es el programa ciberconvivencia-voluntarios (Maristas Centro Cultural Vallisoletano, 2012); el programa para la mejora de la ciberconvivencia en los Institutos de Educación Secundaria (Junta de Andalucía y IES San José, 2014), el programa Ciberconvivencia para $4^{\circ}$ grado de Educación Secundaria Obligatoria (Agustinas Valladolid, 2015) y la iniciativa ciberconvivencia por unas pantallas sanas (Gobierno de Aragón y Pantallas Sanas, 2016).

A pesar de existir estas propuestas ninguna de ellas ha reportado datos que demuestren su efectividad, ya sea por medio de la evaluación directa la ciberconvivencia que presenten los individuos a los que fue dirigido el programa o bien probar la efectividad de estos por medio de prevalencia de cyberbullying presentada antes y después de su implementación; es por esto que el presente artículo busca exponer los ejes de un programa basado en la ciberconvivencia para la prevención del cyberbullying, así como la demostración de su efectividad de manera empírica.

\section{Método}

El estudio fue realizado por medio de un diseño tipo cuasi experimental pretest-postest, con dos grupos, control y experimental, con el objetivo de evaluar la efectividad de un programa para prevenir el cyberbullying basado en la ciberconvivencia.

\section{Muestra}

El universo de estudio se conformó por estudiantes de nivel medio superior de la Universidad Autónoma del Estado de México, del primero, tercero y quinto semestre de bachillerato en el ciclo escolar 2016-2017 en el turno matutino; de la cual se obtuvo una muestra intencional no probabilística conformada por 88 alumnos cuya edad oscila en el rango de 15 a 18 años; 44 de ellos conformaron el grupo experimental donde el 56.82\% fueron mujeres; y los otros 44 alumnos formaron parte del grupo control donde la cantidad de mujeres correspondía a un 52.27\%. Estos datos, como se observa, muestran que los participantes representan adecuadamente a la población de este nivel educativo, y por lo tanto es significativa para poder realizar el estudio.

\section{Instrumento}

Para evaluar la efectividad del programa y determinar la prevalencia de cyberbullying que existía en los grupos antes y después de su implementación se aplicó una adaptación del Cyberbullying Questionnarie (CBQ) de Gámez-Guadix, Villa-George, y Calvete (2014), el cual 
está que conformado por tres subescalas; dos de ellas con 14 reactivos una para medir la perpetración y otra para medir victimización; la tercer subescala cuenta con 5 reactivos para medir el índice de justificación que se tiene del cyberbullying.

Los reactivos van encaminados a preguntar con qué frecuencia han realizado en los últimos 2 meses una serie de conductas de Cyberbullying (perpetración) y con qué frecuencia han sufrido estas conductas (victimización). Para el caso de la subescala de justificación los reactivos hacen alusión a la defensa de realizar actos de cyberbullying.

La adaptación del CBQ fue sometido a un pilotaje que arrojó un Alfa de Cronbach general de 0.939. En cuanto a la fiabilidad por subescala el instrumento presento aperpetración $=0.946$, avictimización $=0.801$ y ajustificación $=0.895$, por lo cual se consideró que su consistencia interna era adecuada para llevar acabo las mediciones del pretest y postest.

\section{Procedimiento}

En cuanto al procedimiento que se siguió para el desarrollo de este trabajo se resume en los siguientes pasos:

1. Diseño del programa de ciberconvivencia. Se basó en la información sobre el tema siendo su objetivo general lograr que los participantes utilicen de manera adecuada los comportamientos, valores, normas y emociones que comprenden las relaciones interpersonales en entornos virtuales con su red de iguales, profesores y familia.

El programa consta de ocho sesiones de una hora de duración; estas estuvieron planificadas para aplicarse una semanalmente y se encuentran divididas en 3 ejes temáticos, a) eje de vida cotidiana en la red (cuatro sesiones); b) eje de normas en los entornos virtuales (tres sesiones) y c) eje de ciberconvivencia pautas para mantener una interacción armónica en los entornos virtuales (una sesión de cierre)

2. Adaptación del instrumento CBQ y muestreo intencional no probabilístico, esto debido al horario escolar de los estudiantes y a que se necesitaba trabajar con el grupo completo, eligiendo para el grupo experimental un grupo de tercer semestre el cual disponía de una hora libre, siendo este horario aprovechado para la aplicación del programa; y para el caso del grupo control igualmente se seleccionó a un grupo de iguales características (tercer semestre) a quien se le aplicó únicamente el cuestionario mencionado.

3. Aplicación del programa de ciberconvivencia. Se procedió a trabajar con los alumnos. Seis de las sesiones se llevaron a cabo en una sala de cómputo, contando cada estudiante con una computadora con acceso a internet. Se realizaron dinámicas de trabajo a través de las TIC, donde los participantes revisaron diversos materiales y actividades por medio de un grupo cerrado en la red social Facebook; esto permitió que la interacción fuera presencial con retroalimentación personalizada por parte del responsable del programa. Las dos sesiones restantes se llevaron a cabo en un auditorio donde expertos en el tema sobre el uso de las TIC y relaciones interpersonales presentaron conferencias dirigidas a los alumnos. Una vez terminada la aplicación del programa en el grupo experimental se esperaron 2 semanas para la aplicación del postest en ambos grupos (control y experimental). 


\section{Análisis de datos}

La aplicación de pretest-postest se hizo mediante la reproducción impresa del instrumento CBQ, creando una base de datos en el Statistical Package for the Social Sciences 19 (SPSS). Se utilizando análisis estadísticos no paramétricos como la prueba de rangos de Wilcoxon y la prueba $U$ de Mann-Whitney. Esto es debido a que no se presentó una distribución normal de los resultados en la muestra.

\section{Resultados}

A continuación, se muestran los hallazgos encontrados en el grupo experimental y de control antes y después de la aplicación del programa de ciberconvivencia.

En primer lugar, respecto a la diferencia existente entre el grupo control y el grupo experimental se realizó la prueba de $U$ de Mann-Whitney para la cual se tomó un nivel de significancia del .05, Los resultados obtenidos en este análisis se muestran en la Tabla1.

Tabla1. U de Mann-Whitney Diferencias entre Grupo Control y Experimental Pretest

\begin{tabular}{cccc}
\hline Subescala & $U$ & $z$ & $p$ \\
\hline Perpetración & 945.500 & -.597 & .550 \\
Victimización & 880.000 & -1.682 & .093 \\
Justificación & 856.500 & -1.300 & .193 \\
\hline
\end{tabular}

En segundo lugar, en cuanto a la implementación del programa se analizaron las diferencias presentadas entre el pretest y postest de ambos grupos por medio de la prueba de rangos de Wilcoxon tomando un nivel de significancia del .05, los resultados obtenidos de dicho análisis se muestran en la Tabla 2.

Tabla2. Wilcoxon Diferencias entre Pretest-Postest en los Grupo Control y Experimental

\begin{tabular}{lcccc}
\hline \multirow{2}{*}{ Subescala } & \multicolumn{2}{c}{ Grupo Control } & \multicolumn{2}{c}{ Grupo Experimental } \\
\cline { 2 - 5 } & $Z$ & $p$ & $z$ & $p$ \\
\hline Perpetración & -1.342 & .180 & -1.000 & .317 \\
Victimización & .000 & 1.000 & -2.236 & $.025^{*}$ \\
Justificación & -.676 & .499 & -2.264 & $.024^{*}$ \\
\hline
\end{tabular}

$* \mathrm{p}<.05$

\section{Discusión}

En primer lugar, si bien se puede resaltar que los resultados obtenidos son favorables en la evaluación del programa de ciberconvivencia para la prevención del cyberbullying; el objetivo de la presente investigación no puede considerarse plenamente alcanzado, los estudiantes mostraron un mejor comportamiento en las relaciones interpersonales que tienen en los entornos virtuales, y con ello, una disminución del acoso escolar cibernético, sin embargo 
los índices desde un comienzo resultaron ser bajos y existieron un serie de dificultades metodológicas.

De acuerdo a la prueba estadística de $U$ de Mann-Whitney no existían diferencias entre el grupo control y el grupo experimental con respecto a las subescalas que conforman el instrumento CBQ antes de la aplicación del programa. Posterior a la aplicación del programa en el grupo experimental la prueba de rangos Wilconxon demostró que no existió un cambio significativo en el grupo control; mientras que en el caso del grupo experimental si hubo una diferencia estadísticamente significativa basada en rangos negativos para las subescalas de victimización y justificación del cyberbullying, lo que indica que las medianas presentadas en el postest por parte del grupo experimental fueron menores a las que presentaron en el prestest.

De igual forma se tiene en cuenta que al ser la primera aplicación formal que se realizó del programa de ciberconvivencia en el nivel medio superior de la UAEMex, se optó por una muestra pequeña para corroborar si el diseño de este llegaba a tener impacto real en la prevención del cyberbullying y posterior realizar aplicación a mayor escala como las presentadas por programas como ConRed (Del Rey, Casas y Ortega, 2012) y el Cyberprogram 2.0 (Garaigordobil y Martínez-Valderrey, 2015) para así someterlo a prueba con estadística paramétrica. Si bien el programa demostró efectividad en la muestra esto solo es un primer paso. Aún está sujeto a mejoras y plantear por qué solo existieron diferencias en dos de las tres subescalas.

Otra dificultad a tomar en cuenta es que si bien se trata de un programa basado de ciberconvivencia cabe aclarar que al abordar los temas con el grupo experimental fue necesario complementar la información daba sobre ciberconvivencia con datos referentes a ciudadanía digital; esto debido a que la ciudadanía digital abarca la necesidad de formar normas de convivencia en los entornos virtuales pero de una manera más amplia; ambos conceptos coinciden en aun no estar bien delimitados lo cual dificulta el poder abordarlos de manera concisa. Así también las condiciones de la aplicación del programa dan paso paso a futuras mejoras de las condiciones de aplicación y cuidar aspectos metodológicos en lo que respecta al muestreo.

A pesar de todo lo mencionado el presente estudio comprueba la premisa planteada de que al educar a los menores en el uso adecuado y responsable de las TIC por medio de aspectos de la ciudadanía digital y ciberconvivencia, se crea un mejor ambiente en los entornos virtuales, lo que lleva a disminuir problemáticas y riesgos de violencia en estos (FloresFernández, 2009; Flores-Fernández, 2010; Ortega, 2011; Santander, 2012; Santander, 2013; Ortega, Casas y Del rey, 2014; Ortega, 2015).

\section{Conclusiones}

La problemática del cyberbullying, a pesar de que diferentes estudios indican que está yendo en aumento, es un tema poco abordado por la agenda de los investigadores, y más aún cuando se trata de generar alternativas de prevención e intervención susceptibles de ser probadas en cuanto a su eficacia.

En el contexto estudiado se han realizado diferentes investigaciones referentes al fenómeno del acoso escolar, entre las que destacamos las siguientes: 
En primer lugar, se llevó a cabo un "Diagnóstico de la intimidación y agresión en los Planteles del Nivel Medio Superior de la Universidad Autónoma del Estado de México" realizado en el año 2012 en una muestra de 12,993 alumnos distribuidos en los nueve planteles de la Escuela Preparatoria de la UAEMex donde se concluyó que "los alumnos no perciben agresión en sus plateles (97\%), y de hecho, la agresión percibida se presenta en un porcentaje bastante bajo (3\%)" (Ibidem,73). A través de este instrumento se midió también el ciberbullying, identificando en un $4.9 \%$, mostrando la existencia en índices muy bajos de este tipo de agresiones.

En segundo lugar, y gracias a este primer acercamiento, se identificó la necesidad de realizar una investigación específica para el ciberbullying. En 2013, se desarrolló el proyecto "Ambientes Virtuales, Aprendizaje y Violencia" que contó con una muestra de 798 estudiantes que fueron encuestados y 300 narraciones de estudiantes involucrados en alguno de los roles del ciberbullyin. Los resultados arrojaron un índice mayor (21\%) destando el método mixto que permitió comprender la experiencia subjetiva de la comunidad universitaria sobre la falta de elementos legales que sancionen este tipo de causas (Morales, Serrano y Santos, 2016).

En tercer lugar, se llevó a cabo un estudio de comparación respecto al género y el rol de víctima y victimario, donde se destaca que las mujeres son más activas en el rol de víctimarias y los hombres son en su mayoría, víctimas (Serrano y Morales, 2015).

En cuarto lugar, se realizó una caracterización a los estudiantes que se involucran en episodios de bullying y ciberbullying identificando los principales roles (Jiménez,2016), encontrando que la mayoría se ubica en el rol de no involucrados $(60.8 \%)$ de un total de 860 estudiantes. El rol de espectador presentó un $6.7 \%$, el de víctima un $2.2 \%$, el víctima-agresor $0.7 \%$ y para aquellos que tienen un rol activo en ciberbullying en un $50.8 \%$ (Jimémez, 2016:84-86).

Finalmente se detectaron las habilidades digitales de los estudiantes, encontrando que éstos no cuentan con un manejo de adecuado de ciudadanía digital y manejo de emociones (Martinez-Vilchis, et. al., 2015).

Todas estas investigaciones previas fundamentan la idea del diseño e implementación de un programa de prevención basado en ciberconvivencia.

En cuanto a su eficacia, éste programa presentó resultados positivos, sin embargo, es necesario realizar nuevas pruebas en poblaciones mucho mayores para poder garantizar su total efectividad como método de prevención secundaria. Por ello, se tiene la intención de volver a implementar el programa de ciberconvivencia, pero ahora en un número mucho mayor de participantes, además de contar con más horas de exposición al mismo, lo cual lleve al rediseño de actividades para un mejor dinamismo en las sesiones y ampliar las temáticas que se abordan.

La intención de continuar con este tipo de estudios es la de garantizar que este tipo de programa es confiable como forma de prevención secundaria y buscar que pueda implementarse de manera obligatoria en todos los planteles del nivel medio superior de la UAEMex. 


\section{Referencias}

Aftab, P. (2010). What is cyberbullying, exactly? Recuperado de $\mathrm{http} / / /$ stopcyberbullying.org/what_is_cyberbullying_exactly.html

Agustinas Valladolid. (2015). Programa Ciberconvivencia (4 ESO) Una iniciativa de voluntariado para el crecimiento personal y la mejora de la convivencia en el centro. Recuperado el 01 de marzo de 2017 desde: http://agustinassecundaria.blogspot.mx/2015/10/programa-ciberconvivencia.html

AMIPCI, INFOTEC y Elogia. (2016). $12^{\circ}$ Estudio sobre los Hábitos de los Usuarios de Internet en México. Recuperado el 29 de enero de 2016, de https://es.slideshare.net/elogia/estudiode-hbitos-de-los-usuarios-de-internet-en-mxico-2016.

Belloch, C. (s.f). Recursos Tecnológicos (TIC). Valencia: Unidad de Tecnología Educativa, Universidad de Valencia. Recuperado el 23 de mayo de 2016 de: http://www.uv.es/bellochc/logopedia/NRTLogo1.pdf.

Belsey, B. (2005). Cyberbullying: An Emerging Threat to the "Always On" Generation. Recuperado el 23 de marzo de 2015 de www.cyberbullying.ca/pdf/Cyberbullying_Article_by_Bill_Belsey.pdf2 de noviembre de 2016 de: http://www.uv.es/bellochc/pedagogia/EVA3.pdf

Buelga, S. y Pons, J. (2012). Agresiones entre Adolescentes a través del Teléfono Móvil y de Internet. Psychosocial Intervention 21(1), 91-101. Recuperado el 23 de mayo de 2015, de http://www.redalyc.org/articuloBasic.oa?id=179824383008

Calmaestra, J. (2011). Cyberbullying: Prevalencia y Características de un nuevo tipo de bullying indirecto. Tesis doctoral. Servicio de Publicaciones de la Universidad de Córdoba. Recuperado el 15 de noviembre de 2016, de http://helvia.uco.es/xmlui/bitstream/handle/10396/5717/9788469490976.pdf?sequence=1

Castro-Santander, A. (2012). La Ciber-Convivencia de los "Screenagers". Revista Meta: Avaliação, 4(12), pp. 314-322. Recuperado de: http://dx.doi.org/10.22347/21752753v4i12.169

Castro-Santander, A. (2013). Formar para la ciberconvivencia Internet y prevención del ciberbullying. Revista Integra Educativa, 6(2), pp. 49-70. Recuperado de http://www.scielo.org.bo/scielo.php?script=sci_arttext\&pid=S199740432013000200004\&1 ng=es\&tlng=es.

Christakis, N. y Fowler, J. (2010): Conectados: El sorprendente poder de las redes sociales y cómo nos afectan. Madrid: Taurus. Recuperado el 19 de noviembre de 2016,de:https://www.academia.edu/2695402/Conectados_El_sorprendente_poder_de_la S_redes_sociales_y_c\% $\%$ C3\%B3mo_nos_afectan

Del Rey, R., Casas, J. A. y Ortega, R. (2012). El programa ConRed, una práctica basada en la evidencia. Comunicar, 39, 129-138. Recuperado de http://www.revistacomunicar.com/index.php?contenido=detalles\&numero=39\&articulo $=39-2012-15$

Del Rey, R. y Ortega, R. (2007). Violencia escolar: claves para comprenderla y afrontarla. Escuela Abierta, 10, 77-89. Recuperado de http://www.researchgate.net/profile/Ortega_Rosario/publication/28203713_Violencia_e scolar_claves_para_comprenderla_y_afrontarla/links/0912f50b6914cbf02e-000000.pdf 
Del Río, J., Bringué, X., Sádaba, C. y González, D. (2009). Cyberbullying: un análisis comparativo en estudiantes de Argentina, Brasil, Chile, Colombia, México, Perú y Venezuela. Paper presentado al V Congrés Internacional Comunicació I Realitat, Barcelona.

Flores-Fernández, J. (2009). Uso seguro de Internet y ciudadanía digital responsable. Recuperado el 22 de noviembre de 2016 de: http://www.pantallasamigas.net/proteccioninfancia-consejos-articulos/uso-seguro-de-internet-y-ciudadania-digitalresponsable.shtm.

Flores-Fernández, J. (2010). Netiqueta Joven para Redes Sociales: ciudadanía digital y ciberconvivencia. Recuperado el 22 de enero de 2017 desde. http://www.pantallasamigas.net/proteccion-infancia-consejos-articulos/netiqueta-jovenpara-redes-sociales-ciudadania-digital-y-ciberconvivencia.shtm

Fundación UNAM. (2013). Ciudadanía digital y competencias digitales. Qué son y qué significan. Recuperado el 27 de enero de 2016 desde: http://www.fundacionunam.org.mx/mi-tecnologia/ciudadania-digital-y-competenciasdigitales-que-son-y-que-significan/

Galindo, J. (2009). Ciudadanía digital. Signo y Pensamiento, XXVIII (54) pp. 164-173. Recuperado de http://www.redalyc.org/articulo.oa?id=86011409011

Gámez-Guadix, M., Villa-George, F. y Calvete E. (2014). Psychometric Properties of the Cyberbullying Questionnaire (CBQ) Among Mexican Adolescents. Violence and Victims, Volume 29, Number 2, pp. 232-247. Recuperado el 26 de Noviembre de 2015 de: http://connection.ebscohost.com/c/articles/95534592/psychometric-propertiescyberbullying-questionnaire-cbq-among-mexican-adolescents

Garaigordobil, M. y Martínez-Valderrey, V. (2015). Effects of Cyberprogram 2.0 on "face-toface" bullying, cyberbullying, and empathy. Psicothema, 20(1), 45-51. Recuperado de http://www.psicothema.com/pdf/4234.pdf

Garaigordobil, M. (2011). Prevalencia y consecuencias del cyberbullying: una revisión. International. Journal of Psychology and Psychological Therapy 11(2), pp. 233-255. Recuperado el 02 de septiembre de 2016, de http://www.federicofroebel.org/secondary/presentacion-redes/prevalencia-yconsecuencias-del-cyberbullying.pdf

García-Maldonado, G., Martínez-Salazar, G.J., Saldívar-González, A.H., SánchezNuncio, R., Martínez-Perales, M., Barrientos-Gómez, M.C. (2012). Factores de riesgo y consecuencias del cyberbullying en un grupo de adolescentes: Asociación con bullying tradicional. Boletín médico del Hospital Infantil de México, 69(6), 463-474. Recuperado de http://www.scielo.org.mx/scielo.php?script=sci_arttext\&pid=S1665$11462012000600007 \& \operatorname{lng}=$ es\&tlng=es.

Gobierno de Aragón y Pantallas Sanas. (2016). Ciberconvivencia por unas pantallas sanas. Recuperado el 01 de marzo de 2017 desde: http://pantallassanas.blogspot.mx/p/basesciberconvivencia-2016.html.

Iab México, Televisa.com y Millward Brown. (2016). Estudio de consumo de medios entre internautas mexicanos, 8va. Edición. Recuperado el 29 de enero de 2016, de http://es.slideshare.net/iabmexico/estudio-consumo-medios-internautas-mexico 
Jiménez, K (2016). Caracterización de los adolescentes involucrados en el bullying. (Tesis de Maestría). México: Universidad Autónoma del Estado de México.

Junta de Andalucía y IES San José, (2014). Programa para la mejora de la ciberconvivencia en el IES San José. Recuperado el 01 de marzo de 2017 desde: https://es.slideshare.net/DavidRomeroMartn/presentacin-programa-mejoraciberconvivencia-34165782

Junta de Castilla y León, (2011). Uso Inteligente de las Nuevas Tecnologías: Consejos Prácticos. España Recuperado el 23 de marzo de 2015 desde: http://www.etnassoft.com/biblioteca/uso-inteligente-de-las-nuevas-tecnologias/

Kerlinger, F. (2002). Investigación del comportamiento. (edi. 4a) México: McGraw-Hill.

Kowalski, R., Limber, S. y Agatston, P. (2008). Cyber Bullying: El acoso escolar en la era digital. Urduliz: Desclée De Brouwer.

Lucio, L.A. (2009). Agresores escolares en el ciberespacio; el cyberbullying en preparatorias mexicanas. Trabajo presentando en XI Asamblea General de ALAFEC. GuayaquilEcuador, 22 al 25 de septiembre (paper).

Maristas Centro Cultural Vallisoletano. (2012). Desarrollo de las competencias básicas. Programa CiberConVivencia-voluntarios. Recuperado el 01 de marzo de 2017 desde: http://orientacionccv.blogspot.mx/2012/03/desarrollo-de-las-competencias-basicas.html

Martínez-Vilchis, R., Pozas, J., Jiménez-Arriaga, K., Morales, T., Miranda, D. A., Delgado, M. E. y Cuenca, V. (2015). Prevención de la violencia escolar cara a cara y virtual en bachillerato. Psychology, Society, \& Education, 7(2), 201-212. Recuperado de http://www.psye.org/articulos/Martinez.pdf

Meneses, M. (2012). Ciudadanía digital: Competencia del individuo en el siglo XXI. Recuperado el 26 de febrero de 2016 desde: http://www.itesm.mx/wps/wcm/connect/snc/portal+informativo/opinion+y+analisis/firm as/dra.+maria+elena+meneses+rocha/op(10may12)mariaelenameneses

Miranda, D.; Serrano, J., Morales, T., Montes de Oca J., Reynoso B. "Agresión y Acoso en el Nivel Medio Superior: El caso de la Universidad Autónoma del Estado de México" En: Pedroza, F:J. y Aguilera S. J.[Coords] (2013) La Construcción de identidades Agresoras: el acoso escolar en México. México: CONACULTA. 73-98.

Morales, T. y Serrano C. (2014) Ra Xi,hai. El mundo, el universo o la vida. Vol 10, núm 4, julio-diciembre, Universidad Autónoma Indígena de México. P. 235-262.

Morales, T., Serrano, C., y Santos, A., (2016). Cyberbullying, acoso cibernético y delitos invisibles. Experiencias Psicopedagógicas. México: Universidad Autónoma del Estado de México.

Moravec, J. (2011). Desde la sociedad 1.0 a la sociedad 3.0. En Cobo, C y Moravec, J. (2011). Aprendizaje Invisible: hacia una nueva ecología de la educación. Col•lecció Transmedia XXI. Laboratori de Mitjans Interactius / Publicacions i Edicions de la Universitat de Barcelona. Barcelona

Moreno, J. (2001), Comportamiento antisocial en los centros escolares: una visión desde Europa. Revista Iberoamérica de Educación, Núm.18. Recuperado el 20 de noviembre de 2016 de: http://www.oei.es/historico/oeivirt/rie18a09.htm

Ortega, R. (2015). Convivencia y ciberconvivencia: Un modelo educativo para la prevención del acoso y el ciberacoso escolar. España: Antonio Machado Libros. 
Ortega R., Casas J., Del Rey R. (2014). Hacia el constructo Ciberconvivencia. Infancia y Aprendizaje: Journal for the Study of Education and Development. 37 (3). pp. 602-608. Recuperado el 13 de mayo de 2015 de: http://www.tandfonline.com/doi/abs/10.1080/02103702.2014.957537

Ortega R., Del Rey, R. y Sánchez, V. (2011). Nuevas Dimensiones de la convivencia escolar. Ciberconducta y Relaciones en red: Ciberconvivencia. Córdoba: Laboratorio de estudios sobre Convivencia y prevención de la violencia. Universidad de Córdoba. Dpto. de Psicología.

Palladino, B.E., Nocentini, A., \& Menesini, E. (2012). Online and offline peer led models against bullying and cyberbullying. Psicothema, 24, 634-639. Recuperado de www.psicothema.com/pdf/4065.pdf

Serrano, C. y Morales, T. (2015) "Violencia virtual: ciberbullying, acoso y género". En: Vélez, G. y Luna, A. [Coords.] Violencia de Género. Escenarios y quehaceres pendientes. México: Universidad Autónoma del Estado de México. 71-100.

Smith, P. K., Mahdavi, J., Carvalho, M. y Tippet, N. (2006). An investigation into cyberbullying, its forms, awareness and impact, and the relationship between age and gender in cyberbullying. A Report to the Anti-Bullying Alliance. Recuperado el 26 de noviembre de 2015 de: http://webarchive.nationalarchives.gov.uk/ 20130401151715/http://www.education.gov.uk/publications/eOrderingDownload/RBX0 3-06.pdf

Vernieri, M.J, (2010). Violencia escolar: ¿se puede hacer algo? Buenos Aires. Editorial Bonum. Willard, N. (2005). Cyberbullying and Cyberthreats. Trabajo presentado en la Conferencia Nacional del Center for Safe and Responsible Use of the Internet, Washington, 15 de agosto. Recuperado el 24 de mayo de 2015 de bcloud.marinschools.org/SafeSchools/Documents/BP-CyberBandT.pdf. 